ESAIM: PROCEEDINGS, March 2012, Vol. 35, p. 46-58

Fédération Denis Poisson (Orléans-Tours) et E. Trélat (UPMC), Editors

\title{
OSCILLATORY LIMITS WITH VARYING SPECTRUM
}

\author{
EMmanUEL GRENIER ${ }^{1}$
}

\begin{abstract}
High time frequency oscillations occur in many different physical cases: slightly compressible fluids, almost quasineutral plasmas, small electron mass approximation .... In many case, small parameters arise in fluids mechanics or plasma physics, leading to these oscillations as the small parameter goes to zero. The aim of this note is to detail how to obtain formal expansions and to give some indications on how to justify them.

Résumé. De fortes oscillations en temps apparaissent dans de multiples situations physiques: fluides faiblement compressibles, plasmas quasineutres, approximation de masse nulle pour les électrons ... Dans de nombreux cas, de petits paramètres apparaissent en mécanique des fluides et des plasmas, conduisant à ces oscillations quand les petits paramètres tendent vers 0 . L'objectif de cette note est de détailler comment obtenir des développements formels et de donner quelques indications sur comment les justifier.
\end{abstract}

\section{INTRODUCTION}

In this note we will discuss three different physical cases: the incompressible limit of barotropic fluids, the incompressible limit of inhomogeneous fluids and the massless limit in plasma physics. In all cases we will detail the formal analysis and give indications on rigorous results.

Oscillatory limits are characterized by the existence of two time scales: a fast time scale which goes to 0 as the small parameter goes to 0 , linked to fast time oscillations or wave propagations, and a slow time scale, of order $O(1)$. Large oscillations occur in the system, with very high frequency. If the domain is the whole space, in many case these oscillations escape to infinity, and leave any compact within a vanishing time. In this case, after an initial time layer, oscillations disappear, and the limit is no longer singular. If on the contrary the domain is periodic or bounded, the oscillations have no way to escape and they remain forever. They interact with themselves and may alter the weak limit of the solutions. Solutions converge only weakly.

The most classical oscillatory limit is the incompressible limit of barotropic flows. The starting point is the Euler equations for compressible barotropic fluids. The small parameter is then the Mach number, ratio of the speed of the fluid to the speed of sound. As the Mach number goes to 0, solutions of the barotropic system go weakly to solutions of the classical Euler equations for incompressible fluids. The difference between the solutions of the barotropic system and the limit solution is a sound wave which has a very high speed $(1 /$ Mach $)$. In the whole space, the sound wave goes to infinity, leading to strong convergence in any compact in space, after an initial time layer. On the contrary, in the periodic or bounded case, the sound waves stay forever and the convergence is only weak because of high time frequency oscillations.

This case is the simplest case since the weak limit decouples from the oscillations. Moreover the oscillations satisfy the classical wave equation which is easily solved using Fourier transform or spectral theorem on the

1 Unité de Mathématiques Pures et Appliquées, CNRS UMR 5669 and INRIA Rhône Alpes, 46 allée d'Italie, 69364, Ecole Normale Supérieure de Lyon

(C) EDP Sciences, SMAI 2012 
Laplace operator. The spectrum of the oscillations is fixed and does not depend on the limit solution. Moreover the limit equation is a variant of Euler equations for which we have local in time existence of smooth solutions. This limit is well known since the works of S. Klainerman, S. Majda, S. Schochet, S. Ukai and many others.

A second classical example is the incompressible limit for inhomogeneous fluids, where the fluid is described by its concentration, velocity and entropy $S$. The limit equations are the Euler equations for inhomogeneous incompressible fluids. In this case the problem is widely open, because of several difficulties which will be discussed in this note. First the limit equation is not decoupled from the oscillations equations, and is even singular (not Lipschitz). Second the wave equations depends on time through the limit entropy. Its spectrum depends on time, and eigenvalue crossings may happen.... In this note we will detail the formal analysis and give some preliminary results on the spectral aspects of this limit.

The third example is the massless limit for a coupled systems of Euler Poisson equations (one on electrons, the other on ions). As the ratio electronic mass / ionic mass goes to 0, electronic oscillations appear. These oscillations are described by a time and space variant of Klein Gordon equations. The corresponding wave operator is skew adjoint in a scalar product which depends on the solution itself. Again its spectral analysis is delicate and only preliminary results will be given here. However the limit equation is very simple, which allows "if" theorems: under strong spectral assumptions we can get justify the formal analysis for almost any initial data. Namely for almost any initial data the limit avoids double eigenvalues and avoids resonances.

\section{A First EXAMPle: COMPRESSIBLE BAROTROPIC FLUIDS}

\subsection{Formal analysis}

Introduction:

Let us begin by the simplest and most classical case: the low Mach number limit, or incompressible limit of barotropic fluids. Motions of water or air around vehicles or obstacles are often modeled by Navier Stokes or Euler equations, assuming a barotropic pressure law (namely: the pressure depends on the density only), which leads to the following system

$$
\begin{gathered}
\partial_{t} \rho+\nabla \cdot(\rho u)=0, \\
\partial_{t} u+\nabla(u \otimes u)+\nabla p(\rho)=0 .
\end{gathered}
$$

We will consider this equation in a periodic domain in dimension 2 or 3 (the dimension plays no rule in the sequel). In these equations, $u(t, x)$ is the velocity (vector of $\mathbb{R}^{2}$ or $\left.\mathbb{R}^{3}\right), \rho$ is a scalar (density) and $p(t, x)$ a given smooth increasing function of $\rho(t, x)$ (pressure). We assume that the pressure only depends on the density (so called barotropic fluid).

There are different ways to introduce a small parameter in this system. A first approach is to assume that $p$ depends rapidly on $\rho$ and to assume that $p^{\prime}(\rho)$ is of order $\varepsilon^{-2}$. This leads to the introduction of a $\varepsilon^{-2}$ in front of $\nabla p(\rho)$. A second approach is to consider the speed of sound which is of order $\sqrt{p^{\prime}(\rho)}$. However usually the speed of cars or wind is much smaller than the speed of sound (of order $1000 \mathrm{~km} /$ hour). Therefore $u$ is small, of size $\varepsilon$. To see an evolution, $u$ should then be studied on time scales of order $\varepsilon^{-1}$. This leads to the introduction of a small parameter $\varepsilon$

$$
u(t, x)=\varepsilon \hat{u}(\varepsilon t, x), \quad \rho(t, x)=\hat{\rho}(\varepsilon t, x) .
$$

System $(1,2)$ turns to (dropping the "hats")

$$
\begin{gathered}
\partial_{t} \rho+\nabla \cdot(\rho u)=0, \\
\partial_{t} u+\nabla(u \otimes u)+\frac{\nabla p(\rho)}{\varepsilon^{2}}=0 .
\end{gathered}
$$

Physically, as $\varepsilon \rightarrow 0$ we expect that the fluid behaves like an incompressible fluid: $\rho$ is constant and the divergence of $u$ vanishes. By doing this, two constraints appear on the solution $(\nabla \rho=0$ and $\nabla \cdot u=0)$. These 
two constraints may not be satisfied by the initial conditions. In this case sound waves of high speed propagate in the domain. These fast oscillations have a vanishing mean value, which leads to the satisfaction of the limit constraints in average (in a weak sense).

Formal limit: incompressible part

Let us detail these physical insights in mathematical terms. First, at a formal level, when $\varepsilon \rightarrow 0$, we get, using (4)

$$
\nabla p(\rho)=0 .
$$

As $p$ is an increasing function this means that $\rho$ is a constant $\rho(t)$. As its integral (the total mass of the fluid in our domain) is constant, $\rho(t, x)$ is a constant $\rho_{0}$. Up to a normalization (change the unit of mass such that the total mass of the fluid is 1 ), we may assume that $\rho_{0}=1$

$$
\rho=1 .
$$

Moreover, using (4) we get

$$
\nabla \cdot u=0
$$

and, noting that the formal limit of a gradient is a gradient,

$$
\partial_{t} u+\nabla(u \otimes u)+\nabla q=0 .
$$

for some new pressure $q$. We thus recover the classical Euler equations for incompressible fluids, namely $(6,7,8)$.

Waves

However the initial data of $(4,5)$ may not always satisfy $(6,7)$. In this case $(7,8)$ is not sufficient to completly describe the limiting process and we have to describe $\rho-1$ and $\nabla . u$. To do this we introduce

$$
\psi=\frac{\rho-1}{\varepsilon}
$$

in order to "equilibrate" $(4,5)$. We get

$$
\begin{gathered}
\partial_{t} \psi+\nabla \cdot(\psi u)+\frac{\nabla \cdot u}{\varepsilon}=0, \\
\partial_{t} u+\nabla(u \otimes u)+\frac{p^{\prime}(1)}{\varepsilon} \nabla \psi+\nabla h_{1}(\psi)+\varepsilon \nabla h_{2}^{\varepsilon}(\psi)=0,
\end{gathered}
$$

where

$$
h_{1}(\psi)=\frac{p^{\prime \prime}(1)}{2} \nabla \psi^{2},
$$

and

$$
h_{2}^{\varepsilon}(\psi)=\varepsilon^{-1}\left(p(1+\varepsilon \psi)-p(1)-\varepsilon p^{\prime}(1) \psi-\frac{p^{\prime \prime}(1)}{2} \varepsilon^{2} \psi^{2}\right) .
$$

Note that $h_{2}^{\varepsilon}$ is bounded uniformly in $\varepsilon$. The singular part of this system is of the form $\varepsilon^{-1} \mathcal{A}$, where

$$
\mathcal{A}\left(\begin{array}{c}
\psi \\
u
\end{array}\right)=\left(\begin{array}{cc}
0 & \nabla \cdot u \\
p^{\prime}(1) \nabla \psi & 0
\end{array}\right) .
$$

If we look at $(9,10)$ in small time, by making the change of variable $T=t / \varepsilon$ (the reverse change of time scale of (3)) and if we keep the leading terms we get the classical sound wave equation

$$
\partial_{t}(\psi, u)+\mathcal{A}(\psi, u)=0
$$


or

$$
\partial_{t t} \psi-p^{\prime}(1) \Delta \psi=0
$$

We will prove that the solution of $(4,5)$ is asymptotically the sum of a solution of the limit system $(6,7,8)$ and of a solution of the wave equation (with a varying profile). There are many different ways to introduce this decomposition. Here we will follow the approach of S. Schochet [13]. Let $\mathcal{L}$ be the resolvent of $(12): \mathcal{L}(t)\left(\psi_{0}, u_{0}\right)$ is the solution of $(12)$ with initial data $\left(\psi_{0}, u_{0}\right)$ at $t=0$. Note that $\mathcal{L}$ can be explicitly computed using Fourier analysis in the periodic case.

\section{Conjugated equation}

We introduce the conjugated unknown

$$
v=\mathcal{L}\left(-\varepsilon^{-1} t\right)(\psi(t), u(t))
$$

which is designed to kill the $\varepsilon^{-1} \mathcal{A}$ term in $(9,10)$. We will denote the components of $v$ by $v=\left(v_{\psi}, v_{u}\right)$. We have

$$
\partial_{t} v+\mathcal{L}\left(-\varepsilon^{-1} t\right) \mathcal{Q}\left(\mathcal{L}\left(\varepsilon^{-1} t\right) v, \mathcal{L}\left(\varepsilon^{-1} t\right) v\right)=0
$$

where $\mathcal{Q}$ collects all the non linear terms

$$
\begin{gathered}
\mathcal{Q}(v, v)=\left(\begin{array}{c}
\nabla \cdot\left(v_{\psi} v_{u}\right) \\
\nabla\left(v_{u} \otimes v_{u}\right)+\nabla h_{1}\left(v_{\psi}\right)
\end{array}\right)+\varepsilon\left(\begin{array}{c}
0 \\
\nabla h_{2}^{\varepsilon}\left(v_{\psi}\right)
\end{array}\right) \\
=\mathcal{Q}_{1}(v, v)+\varepsilon \mathcal{Q}_{2}(v, v) .
\end{gathered}
$$

Now $\partial_{t} v$ is formally bounded and the limit $\varepsilon \rightarrow 0$ is no longer singular. To pass the limit in (14) we need to detail $\mathcal{L}$. Let $\Pi$ be the projector of $v$ on the divergence free part of $v_{u}$

$$
\Pi v=\left(0, \Pi_{L} v_{u}\right)
$$

where $\Pi_{L}$ is the Leray projector on divergence free vector field. Note that

$$
\operatorname{ker} \mathcal{A}=\left\{\left(0, v_{u}\right) \quad \mid \quad \Pi_{L} v_{u}=0\right\}
$$

We recall that for an arbitrary vector field $v$,

$$
\Pi_{L} v=v-\nabla \Delta^{-1} \nabla \cdot v
$$

in such a way that $\nabla \cdot \Pi_{L} u v=0$.

Note that

$$
\mathcal{L}(t) \Pi v=\Pi v
$$

for any $v$ and any $t$. To compute $\mathcal{L}(1-\Pi)$ we introduce the eigenvalues $\lambda_{k}$ with related eigenspaces $P_{k}$. Let $\Pi_{k}$ be the projector on $P_{k}$. Then

$$
\mathcal{L}(t) v_{0}=\Pi v+\sum_{k \geq 1} e^{i \varepsilon^{-1} \lambda_{k} t} \Pi_{k} v_{0}
$$

Note that $P_{k}$ and $\lambda_{k}$ may be easily computed using Fourier transfom.

\section{Limit equations:}

We use (16) to pass the limit in $\mathcal{Q}_{1}$. After some computations which are detailled below, this leads to the following limiting equations

$$
\partial_{t} \Pi v+(\Pi v \otimes \Pi v)+\nabla P=0
$$




$$
\partial_{t}(1-\Pi v)+\mathcal{Q}_{l}(\Pi v,(1-\Pi v))+\mathcal{Q}_{o}((1-\Pi v),(1-\Pi b))=0 .
$$

Note that (17) are simply Euler equations for incompressible flows, with a limiting pressure $P$. Moreover (18) describes the evolution of the wave part $(1-\Pi) v$, with two terms: $Q_{l}$ which takes into account the interaction with the underlying incompressible limit flow $\Pi v$ and $Q_{o}$ which describes non linear interactions between waves (resonances).

Details of the computations of $(17,18)$

$$
\mathcal{L}\left(-\varepsilon^{-1} t\right) \mathcal{Q}\left(\mathcal{L}\left(\varepsilon^{-1} t\right) v, \mathcal{L}\left(\varepsilon^{-1} t\right) v\right)=\mathcal{L}_{1}+\mathcal{L}_{2}^{\varepsilon}+\mathcal{L}_{3}^{\varepsilon}+\mathcal{L}_{4}^{\varepsilon}
$$

where

$$
\begin{gathered}
\mathcal{L}_{1}=\Pi \mathcal{Q}(\Pi v, \Pi v), \\
\mathcal{L}_{2}^{\varepsilon}=\mathcal{L}\left(-\varepsilon^{-1} t\right) \sum_{k \geq 1} e^{i \varepsilon^{-1} t \lambda_{k}} \mathcal{Q}\left(\Pi v, \Pi_{k} v\right) \\
\mathcal{L}_{3}^{\varepsilon}=\mathcal{L}\left(-\varepsilon^{-1} t\right) \sum_{k \geq 1} e^{i \varepsilon^{-1} t \lambda_{k}} \mathcal{Q}\left(\Pi_{k} v, \Pi v\right) \\
\mathcal{L}_{4}^{\varepsilon}=\mathcal{L}\left(-\varepsilon^{-1} t\right) \sum_{k, l \geq 1} e^{i \varepsilon^{-1} t\left(\lambda_{k}+\lambda_{l}\right)} \mathcal{Q}\left(\Pi_{k} v, \Pi_{l} v\right) .
\end{gathered}
$$

All these terms may be easily explicitly computed using Fourier transform and explicit expressions of $\lambda_{k}$ and $\Pi_{k}$. First $\mathcal{L}_{1}$ is the non linearity of incompressible Euler equations

$$
\mathcal{L}_{1}=\left(\begin{array}{c}
0 \\
\Pi(\Pi v \otimes \Pi v)
\end{array}\right) .
$$

Next

$$
\mathcal{L}_{2}^{\varepsilon}=\sum_{k, l \geq 1} e^{i \varepsilon^{-1} t\left(\lambda_{k}-\lambda_{l}\right)} \Pi_{l} \mathcal{Q}\left(\Pi v, \Pi_{k} v\right) \rightarrow \mathcal{L}_{2}=\sum_{k \geq 1} \Pi_{k} \mathcal{Q}\left(\Pi v, \Pi_{k} v\right)
$$

in a weak sense. Similarly

$$
\mathcal{L}_{3}^{\varepsilon} \rightarrow \mathcal{L}_{3}=\sum_{k \geq 1} \Pi_{k} \mathcal{Q}\left(\Pi_{k} v, \Pi v\right)
$$

and

$$
\mathcal{L}_{4}^{\varepsilon} \rightarrow \mathcal{L}_{4}=\sum_{k, l, m \geq 1, \lambda_{k}+\lambda_{l}=\lambda_{m}} \Pi_{m} \mathcal{Q}\left(\Pi_{k} v, \Pi_{l} v\right)+\Pi \sum_{k, l \geq 1, \lambda_{k}+\lambda_{l}=0} Q\left(\Pi_{k} v, \Pi_{l} v\right) .
$$

The second sum in $\mathcal{L}_{4}$ appears to be a gradient, hence the second term in $\mathcal{L}_{4}$ vanishes and $\mathcal{L}_{4}$ reduces to the first interaction term. We then collect $\mathcal{L}_{2}+\mathcal{L}_{3}$ in $Q_{l}$ and $L_{4}$ in $Q_{o}$.

\subsection{Justification of the formal limit}

The first step to justify the previous formal limit is to get existence of solutions to $(4,5)$ on a time interval $[0, T]$ independent on $\varepsilon$, together with Sobolev type bounds on $\psi^{\varepsilon}$ and $u^{\varepsilon}$. This has been proved by S. Klainerman and A. Majda in [9], [10]. At this point we have Sobolev bounds in spatial variables, but not in time. Hence we have not enough compactness to pass the limit.

The second step is to introduce $v=\mathcal{L}\left(\varepsilon^{-1} t\right)(\psi(t), u(t))$. As $\mathcal{L}$ is an isometry in $H^{s}, v$ is uniformly bounded in $H^{s}$ for $t \leq T$ and uniformly in $\varepsilon$. Moreover using (14), $\partial_{t} v$ is uniformly bounded. Classical compactness results lead to the convergence of $v$.

It is then possible to pass the limit in $\mathcal{Q}_{1}$, which rigorously justify the formal limit. Let us sum up these lines in 
Theorem 2.1. Let $s>d / 2+1$. Let $\rho_{0}^{\varepsilon}$ and $u_{0}^{\varepsilon}$ be a sequence of initial data such that

$$
\left\|u_{0}^{\varepsilon}\right\|_{H^{s}}+\varepsilon^{-1}\left\|\rho_{0}^{\varepsilon}-1\right\|_{H^{s}} \leq C_{0}
$$

for every $0<\varepsilon<1$. Then there exist $T>0, C_{1}$ and solutions $\rho^{\varepsilon}$ and $u^{\varepsilon}$ to $(4,5)$ with initial data $\rho_{0}^{\varepsilon}$ and $u_{0}^{\varepsilon}$ such that

$$
\left\|u^{\varepsilon}\right\|_{L^{\infty}\left([0, T], H^{s}\right)}+\varepsilon^{-1}\left\|\rho^{\varepsilon}-1\right\|_{L^{\infty}\left([0, T], H^{s}\right)} \leq C_{1}
$$

Moreover $u^{\varepsilon}$ converges weakly to a solution $u$ of Euler equations for incompressible fluids, and

$$
v^{\varepsilon}=\mathcal{L}\left(-\varepsilon^{-1} t\right)\left(\varepsilon^{-1}\left(\rho^{\varepsilon}(t)-1\right), u^{\varepsilon}(t)\right)
$$

converge strongly to a solution of $(17,18)$. Moreover $(0, u(t))=\Pi v(t)$.

Note that (19) naturally comes from energy considerations. We will not give more details here and refer to $[13]$.

\subsection{Remarks}

This situation is very particular to the periodic case. In the whole space the conclusion is completly different. As sound waves go to infinity with high speed $(1 / \varepsilon)$, the energy of sound waves goes to 0 exponentially fast (like $\exp (-C t / \varepsilon))$ in any bounded set. Hence after a time initial layer of size $O(\varepsilon)$, all the sound waves disappear, and $\psi \rightarrow 1, \nabla . u \rightarrow 0$ strongly in bounded sets. This has been proved by S. Ukai [15] for classical solutions of Euler equations (see [3] for weak solutions of Navier Stokes equations, using Strichartz type estimates).

When viscosity is added, the situation is similar to the inviscid case in the periodic setting or in the whole space. In a bounded domain however the behavior is very different, since boundary layers appear which kills sound waves (by energy dissipation in the boundary layer), within times of order $\sqrt{\varepsilon}$. We have strong convergence to the limit incompressible equations, after an initial boundary layer (see [4]).

The situation is very similar in the case of rotating fluids. Sounds waves are then replaced by inertial or Rossby waves. We refer to [6], [5], [2] for more details.

\section{A SECOND EXAMPLE: INHOMOGENEOUS FLUIDS}

\subsection{Formal limit}

Introduction:

Let us turn to a more complex case: the incompressible limit of inhomogenenous fluids. This system has been first studied by G. Métivier and S. Schochet in [11], [12]. The starting point is the Euler system for inhomogeneous fluids

$$
\begin{gathered}
\partial_{t} \rho+\nabla \cdot(\rho u)=0, \\
\rho\left(\partial_{t} u+u \cdot \nabla u\right)+\frac{\nabla p}{\varepsilon^{2}}=0, \\
\partial_{t} S+u \cdot \nabla S=0,
\end{gathered}
$$

where $u$ is the velocity of the fluid, $\rho$ its density and $S$ its entropy. Here $p$ is the pressure, which is a function of $\rho$ and $S$, given implicitly through the state law of the fluid

$$
\rho=R(p, S)
$$

where $R$ is a given smooth function. For instance one may consider the isentropic state law

$$
\rho=p^{1 / \gamma} e^{-S / \gamma}
$$


As in the previous section, initially, system $(21,22,23)$ is written with $\varepsilon=1$. The change of variable (3) leads to a factor $1 / \varepsilon^{2}$ in front of the pressure term, and hence leads to $(21,22,23)$.

A very tricky change of variable (G. Métivier, S. Schochet) "equilibrates" the system. Let $q$ be defined by

$$
p=\bar{p} e^{\varepsilon q} .
$$

Then

$$
\begin{gathered}
a\left(\partial_{t} q+u \cdot \nabla q\right)+\varepsilon^{-1} \nabla \cdot u=0 \\
r\left(\partial_{t} u+u \cdot \nabla u\right)+\varepsilon^{-1} \nabla q=0 \\
\partial_{t} S+u \cdot \nabla S=0
\end{gathered}
$$

where $a$ and $r$ are two functions of $\rho$ and $S$.

The first step is to prove the existence of solutions on a fixed time interval $[0, T]$, with Sobolev bounds which are uniform in $\varepsilon$. This delicate point has been solved in [11]. The second step is to pass the limit as $\varepsilon \rightarrow 0$. Let us first detail the formal analysis.

Limit system: incompressible part

Formally as $\varepsilon$ goes to 0 , we formally get

$$
\nabla \cdot u=0
$$

(the limit flow is incompressible) and

$$
\nabla q=0
$$

which means that the pressure $q$ is a constant $\bar{p}$. Moreover, the limit of a gradient being a gradient, we get

$$
r\left(\partial_{t} u+u \cdot \nabla u\right)+\nabla \Pi=0
$$

for some pressure $\Pi$, and

$$
\partial_{t} S+u \cdot \nabla S=0 .
$$

The limit of the state law is simply $\rho=R(\bar{p}, S)$ hence $\rho$ is a function of $S$. Equations $(29,30)$ form the Euler system for inhomogeneous incompressible fluids.

\section{Wave equation}

In this case the wave equation is

$$
\partial_{t}(\sigma, v)=\varepsilon^{-1} \mathcal{A}(\sigma, v)
$$

where

$$
\mathcal{A}=\left(\begin{array}{cc}
0 & a^{-1}(S) \nabla \\
r^{-1}(S) \nabla & 0
\end{array}\right)
$$

or, equivalently, after computation of $a(S)$ and $r(S)$,

$$
\varepsilon^{2} \partial_{t t} \sigma-\nabla \cdot(\phi(S(t, x)) \nabla \sigma)=0
$$

for some function $\phi$. Note that formally $\partial_{t} S$ is bounded, hence (31) is a wave equation with high wave speed and with slowly varying coefficients. Moreover $\nabla \cdot(\phi(S) \nabla$.) (and therefore $\mathcal{A}$ ) is skew adjoint.

\section{Difficulties}

The main difficulty is that now the wave equation depends on the solution itself through $S$. The wave equation involves a fast scale $(O(\varepsilon)$, the speed of sound being $1 / \varepsilon$ here), and a slow scale $(O(1)$, linked to the evolution of $S(t)$ ). The solution of the wave equation will be a sum of highly oscillatory modes (with frequencies of order $O(1 / \varepsilon)$ ), the eigenmodes being slowly varying with time (within times of order $O(1)$ ). This is highly reminiscent 
to an adiabatic limit. Problems arise when eigenvalues of $\mathcal{A}$ cross, since energy may then be exchanged between modes.

\section{Resolvent}

Let $\mathcal{L}^{\varepsilon}\left(t, t^{\prime}\right)$ be the resolvent of $(31): \mathcal{L}^{\varepsilon}\left(t, t^{\prime}\right)\left(\sigma_{0}, v_{0}\right)$ is the solution of $(31)$ with initial condition $\left(\sigma_{0}, v_{0}\right)$. When $S(t, x)$ is a constant $S_{0}$ independent on $t$ and $x, \mathcal{L}^{\varepsilon}$ is easily computed using Fourier transform and is an isomorphism from $H^{s}$ to $H^{s}$ for any $s$.

When $S(x)$ depends on $x$ only and not on $t$, we introduce the eigenvalues $\lambda_{k}(S)$ and eigenspaces $P_{k}(S)$ of $\mathcal{A}$, together with the projector $\Pi$ on the kernel of $\mathcal{A}$, and the projectors $\Pi_{k}(S)$ on $P_{k}(S)$. The resolvent in this case is

$$
\mathcal{L}^{\varepsilon}\left(t, t^{\prime}\right)\left(\sigma_{0}, v_{0}\right)=\Pi\left(\sigma_{0}, v_{0}\right)+\sum_{k \geq 1} e^{i \lambda_{k}(S) t / \varepsilon} \Pi_{k}(S)\left(\sigma_{0}, v_{0}\right) .
$$

In this case $\mathcal{L}^{\varepsilon}$ will be bounded from $H^{s}$ to $H^{s}$, uniformly with respect to $t$ and $\varepsilon$.

In general however $S$ depends on both variables $t$ and $x$. In this case we have no explicit formula for $\mathcal{L}^{\varepsilon}$ and we have only approximate resolvents. The only natural energy estimate is the $L^{2} / H^{1}$ estimate. It is not possible to get uniform estimates in high order Sobolev space, since any differentiation of the equation in $t$ or $x$ leads to an $1 / \varepsilon$ term. The behavior of the solution of (32) may be very complex (energy exchange between modes when eigenvalues collide for instance). To get bounds on $\mathcal{L}^{\varepsilon}$ we need to study in detail the spectrum of $\mathcal{A}$ and try to avoid multiple eigenvalues (except those given by symmetries). In the sequel we will not consider multiplicity created by symmetries in the domain, but focus on the change of multiplicity of simple eigenvalues.

\subsection{Geometry of the eigenvalues}

The main difficulty is that eigenvalues of $\mathcal{A}$ may cross. We therefore define

$$
\Sigma_{j, k}=\left\{S \quad \mid \quad \lambda_{j}(S)=\lambda_{k}(S)\right\}
$$

set of $S$ where the eigenvalues $\lambda_{j}$ and $\lambda_{k}$ coincide. Note that all the $\lambda_{j}(S)$ may be choosen locally in a continuous manner. However it is not possible to choose the projectors $\Pi_{j}$ in a continuous way, since near $\Sigma_{j, k}$, neither $\Pi_{j}$ nor $\Pi_{k}$ are continuous, but only their sum $\Pi_{j}+\Pi_{k}$ (locally, provided $\lambda_{j}$ is not a triple eigenvalue).

An elementary example: two by two symmetric matrices

Let us first consider the very simple case of two by two symmetric matrices, of the form

$$
A=\left(\begin{array}{ll}
a & b \\
b & c
\end{array}\right)
$$

The characteristic polynomial is

$$
X^{2}-(a+c) X+a c-b^{2}
$$

and the eigenvalues are

Hence

$$
\lambda_{ \pm}=\frac{a+c}{2} \pm \frac{\sqrt{(a-c)^{2}+b^{2}}}{2}
$$

$$
\Sigma_{j, k}=\{b=0, a=c\}
$$

is a line in a dimension 3 space. Moreover the eigenvectors only depend on $x-\Pi x$, where $\Pi$ is the projection on $\Sigma_{j, k}$, and the eigenvectors "rotate" when we "rotate" around $\Sigma_{j, k}$.

This situation is general: the set of symmetric matrices is of codimension 2 in the set of matrices. And in an heuristic way: the set of selfadjoint operators is of codimension 2 is the set of operators. We therefore expect

$\Sigma_{j, k}$ is generically of codimension 2 
Let us study $\Sigma_{j, k}$ near a double eigenvalue $\lambda_{j}\left(S_{0}\right)=\lambda_{k}\left(S_{0}\right)$. Assume that near $\Sigma_{j, k}, \lambda_{j}(S)$ and $\lambda_{k}(S)$ are simple, and bounded away from all the other eigenvalues $\lambda_{l}(S)(l \neq j, k)$. Then in the vicinity of $S_{0}$ we get an expansion of $\lambda_{j}$ and $\lambda_{k}$, given by

$$
\begin{gathered}
\lambda_{j}\left(S_{0}+S\right), \lambda_{k}\left(S_{0}+S\right)=\frac{\lambda_{j}\left(S_{0}\right)+\lambda_{k}\left(S_{0}\right)}{2}+\frac{1}{2}\left(\int S\left|\nabla \psi_{j}\right|^{2}+\int S\left|\nabla \psi_{k}\right|^{2}\right) \\
\pm \frac{1}{2} \sqrt{\left(\int S\left|\nabla \psi_{j}\right|^{2}-\int S\left|\nabla \psi_{k}\right|^{2}\right)^{2}+4\left(\int S \nabla \psi_{j} \nabla \psi_{k}\right)^{2}}+O\left(|S|_{H^{s}}^{2}\right) .
\end{gathered}
$$

In particular $\operatorname{Vect}\left(\left|\nabla \psi_{j}\right|^{2}-\left|\nabla \psi_{k}\right|^{2}, \nabla \psi_{j} \nabla \psi_{k}\right)$ is perpendicular to $\Sigma_{j, k}$. Under assumption

$$
\text { (H1) } \quad\left|\nabla \psi_{j}\right|^{2}-\left|\nabla \psi_{k}\right|^{2} \text { and } \quad \nabla \psi_{j} \nabla \psi_{k} \quad \text { are linearly independent, }
$$

$\Sigma_{j, k}$ is locally of codimension 2. More precisely,

Theorem 3.1. Let us assume that near $S_{0} \in \Sigma_{j, k}$ there is no triple eigenvalues, and that $(H 1)$ holds true. Then near $S_{0}, \Sigma_{j, k}$ is a codimension two manifolds.

Let

$$
\Sigma_{2}=\cup_{j \neq k} \Sigma_{j, k}
$$

Theorem 3.2. Let us assume that near $S_{0}$ there is no triple eigenvalues, and that (H1) holds true for every $j$ and $k$. Then near $S_{0}, \Sigma_{2}$ is the union of a finite or infinite number of codimension two manifolds.

Note that $(H 1)$ is a spectral assumption. Up to now we do not whether $(H 1)$ holds true or not. We however believe that $(H 1)$ is generic.

\subsection{Away from double eigenvalues}

Bounds on the resolvent

Let us go back to our wave equation

$$
\partial_{t t} \sigma-\varepsilon^{-2} \nabla \cdot(\phi(S(t, x)) \nabla \sigma)=0,
$$

assuming that $S(t)$ does not cross $\Sigma_{2}$. We expand the solution $\sigma$ on the eigenvectors $\psi_{j}$ and introduce rapidly changing phases

$$
\sigma(t)=\sum_{j} \alpha_{j}(t) \psi_{j}(S(t)) \exp \left(\varepsilon^{-2} \int_{0}^{t} \lambda_{j}(S(t))\right) .
$$

Then

$$
\partial_{t} \alpha_{j}=-\left(\sum_{k} \alpha_{k}(t) \nabla \psi_{k}(S(t)) . S^{\prime}(t) \quad \mid \quad \psi_{j}(S(t))\right)
$$

which is bounded away from $\Sigma_{2}$, with singularities on $\Sigma_{2}$. As a consequence, if $S(t)$ never crosses $\Sigma_{2}$, then one can show that the resolvant $\mathcal{L}^{\varepsilon}$ is a bounded operator from $H^{s}$ to $H^{s}$ uniformly in $\varepsilon$.

\section{Resonances}

Let us define the resonant sets

$$
\Sigma_{j, k, l}=\left\{S \quad \mid \quad \lambda_{j}(S)+\lambda_{k}(S)=\lambda_{l}(S)\right\}
$$

and

$$
\Sigma_{3}=\cup_{j, k, l} \Sigma_{j, k, l}
$$


Heuristically $\Sigma_{j, k, l}$ is of codimension 1 . Namely if $\lambda_{k}(S)$ is a simple eigenvalue, then $\lambda_{k}$ is differentiable in $S$ and

$$
d \lambda_{k}(S) . \tilde{S}=\int\left|\nabla \psi_{k}\right|^{2} \tilde{S}
$$

Hence, provided $\lambda_{j}, \lambda_{k}$ and $\lambda_{l}$ are simple at $S, \Sigma_{j, k, l}$ has a normal at $S$ given by

$$
n_{j, k, l}(S)=\left|\nabla \psi_{j}\right|^{2}+\left|\nabla \psi_{k}\right|^{2}-\left|\nabla \psi_{l}\right|^{2}
$$

if this quantity does not vanish,. In particular $\Sigma_{j, k, l}$ is locally of codimension 1 , provided $n_{j, k, l}(S) \neq 0$.

Theorem 3.3. Let us assume that $\lambda_{j}, \lambda_{k}$ and $\lambda_{l}$ are simple eigenvalues near $S_{0}$. Then locally near $S_{0}$, provided

$$
\left|\nabla \psi_{j}\right|^{2}+\left|\nabla \psi_{k}\right|^{2}-\left|\nabla \psi_{l}\right|^{2} \neq 0
$$

$\Sigma_{j, k, l}$ is a codimension 1 manifold.

Note that (H2) is a spectral assumption. We have no idea whether this holds true or not, or whether this is generic or not.

\subsection{General strategy and open questions}

Let us mention at this point that the justification of the formal limit is widely open. Many difficulties still remain.

The best case occurs when the limit entropy $S(t)$ does not cross $\Sigma_{2}$. In this case, the resolvent $\mathcal{L}^{\varepsilon}$ is uniformly bounded. We can then introduce $(\tilde{q}, \tilde{u})=\mathcal{L}\left(\varepsilon^{-1} t\right)(q, u)$, which is bounded in Sobolev spaces, uniformly in $\varepsilon$, and show that $\partial_{t}(\tilde{q}, \tilde{u})$ is bounded. As a consequence, $(\tilde{q}, \tilde{u})$ converge strongly. It remains then to justify the formal analysis. The main problems lie of course in the nonlinear terms. If $S(t)$ avoids $\Sigma_{3}$ or crosses $\Sigma_{3}$ transversally then the formal limit can be fully justified.

It remains to prove that generically $S(t)$ does not cross $\Sigma_{2}$ and that generically it crosses $\Sigma_{3}$ is a transverse way. Note that $\Sigma_{3}$ being of codimension 1 , generically $S(t)$ crosses it.

The main problem is that the limit equation is precisely singular on $\Sigma_{2}$, and not Lipschitz near $\Sigma_{2}$. Up to now, we are not able to define globally a flow for the limit equation, and therefore we fail to prove almost everywhere results. We can only pass the limit if we a priori know that $S(t)$ does not cross $\Sigma_{2}$ but we are not able to prove that this is true for almost any initial data.

\section{A ThiRd EXAMPle: MASSlESS Limit}

This part has been done in collaboration with Y. Guo and B. Pausader. Let us now study an example coming from plasma physics. We consider a plasma composed of ions and electrons, evolving under the action of an electric field. We follow a fluid description of ions and electrons, which leads to the classical Euler Poisson system

$$
\begin{gathered}
\partial_{t} n_{e}+\nabla \cdot\left(n_{e} v_{e}\right)=0 \\
\eta^{2} n_{e}\left(\partial_{t} v_{e}+v_{e} \cdot \nabla v_{e}\right)=-\nabla n_{e}+n_{e} \nabla V \\
\partial_{t} n_{i}+\nabla \cdot\left(n_{i} v_{i}\right)=0 \\
n_{i}\left(\partial_{t} v_{i}+v_{i} \cdot \nabla v_{i}\right)+\nabla n_{i}+n_{i} \nabla V=0 \\
-\Delta V=n_{i}-n_{e}
\end{gathered}
$$

where

$$
\eta=\frac{m_{e}}{m_{i}}
$$

ratio of the masses of the electron and ions, is a small parameter. We will consider the limit $\eta \rightarrow 0$. In this system, $n_{i}$ and $v_{i}$ are the density and velocity of the ions, $n_{e}$ and $v_{e}$ the density and velocity of the electrons, and $V$ the electric potential. 
Formal limit

As $\eta \rightarrow 0$, formally, $n_{e}, V, v_{i}$ and $n_{i}$ converge to $n_{e}^{0}, V^{0}, v_{i}^{0}$ and $n_{i}^{0}$ with

$$
\nabla n_{e}^{0}=n_{e}^{0} \nabla V^{0}
$$

hence

therefore

$$
n_{e}^{0}=e^{-V^{0}}
$$

$$
\begin{gathered}
\partial_{t} n_{i}^{0}+\nabla \cdot\left(n_{i}^{0} v_{i}^{0}\right)=0, \\
n_{i}\left(\partial_{t} v_{i}^{0}+v_{i}^{0} \cdot \nabla v_{i}^{0}\right)+\nabla n_{i}^{0}+n_{i}^{0} \nabla V^{0}=0, \\
-\Delta V^{0}=n_{i}^{0}-e^{-V^{0}} .
\end{gathered}
$$

Note that the dynamics of the ions is completely decoupled from the dynamics of the electrons. Equations $(40,41,42)$ form an independent system, with locally smooth solutions.

The density of the electrons is driven by the potentiel through (39). It is more delicate to describe the velocity of the electrons. Their average velocity is such that (39) is satisfied, in particular $\nabla \cdot\left(n_{e}^{0} v_{e}^{0}\right)$ is prescribed (and equals $\left.-\partial_{t} n_{e}^{0}\right)$. There is also large oscillations in the velocity, as will be detailed below.

\section{Oscillations}

We will observe oscilations of order $O(1)$ on $v_{e}^{\eta}$ and of order $O(\eta)$ on $n_{e}^{\eta}$ and $V$. To see them we make a change of variables. Let $\tilde{v}_{e}, \tilde{n}_{e}$ and $\tilde{V}$ be defined by

$$
\begin{gathered}
v_{e}^{\eta}=v_{e}^{0}+\tilde{v}_{e}, \\
n_{e}^{\eta}=n_{e}^{0}+\eta \tilde{n}_{e}, \\
V^{\eta}=V^{0}+\eta \tilde{V} .
\end{gathered}
$$

Note that $V_{0}$ is simply the limit potential and $n_{e}^{0}=e^{-V_{0}}$ is completely given by the limit ionic dynamics. We get

where

$$
\partial_{t}\left(\tilde{n}_{e}, \tilde{v}_{e}\right)=\eta^{-1} A\left(n_{e}^{0}(t)\right)\left(\tilde{n}_{e}, \tilde{v}_{e}\right)+\left(S_{1}^{\eta}, S_{2}^{\eta}\right)
$$

$$
A\left(n_{e}^{0}\right)(n, v)=\left(\begin{array}{c}
-\nabla \cdot\left(n_{e}^{0} v\right) \\
-\nabla\left(\frac{n}{n_{e}^{0}}\right)+\nabla \Delta^{-1} n
\end{array}\right)
$$

and where $S_{1}^{\eta}, S_{2}^{\eta}$ are nonlinear terms, given by

$$
\begin{gathered}
S_{1}^{\eta}=-\nabla \cdot\left(\tilde{n}_{e} v_{e}^{0}\right)-\nabla \cdot\left(\tilde{n}_{e} \tilde{v}_{e}\right) \\
S_{2}^{\eta}=-\partial_{t} v_{e}^{0}-\left(v_{e} \cdot \nabla\right) v_{e}-\frac{1}{2} \nabla\left(\frac{\tilde{n}_{e}}{n_{e}^{0}}\right)^{2}-\eta \nabla h\left(n_{e}, \tilde{n}_{e}\right),
\end{gathered}
$$

where

$$
\eta^{3} h=\log \left(n_{e}^{0}+\eta \tilde{n}_{e}\right)-\log \left(n_{e}^{0}\right)-\eta \frac{\tilde{n}_{e}}{n_{e}^{0}}-\frac{\eta^{2}}{2}\left(\frac{\tilde{n}_{e}}{n_{e}^{0}}\right)^{2} .
$$

Note that if $n_{e}^{0}=1$ we recover the classical Klein Gordon equation

$$
\partial_{t t} n+n-\Delta n=0
$$

\section{Existence results}

The first step is to get existence of classical solutions on a fixed time interval $[0, T]$, together with uniform $H^{s}$ bounds as $\eta \rightarrow 0$. This is detailled in [7] where bounds of the form

$$
\left\|n_{i}^{\eta}\right\|_{L^{\infty}\left([0, T], H^{s}\right)}+\left\|v_{i}^{\eta}\right\|_{L^{\infty}\left([0, T], H^{s}\right)}+\left\|V^{\eta}\right\|_{L^{\infty}\left([0, T], H^{s}\right)}
$$


are obtained.

$$
+\left\|\frac{n_{e}^{\eta}-e^{-V^{\eta}}}{\sqrt{\eta}}\right\|_{L^{\infty}\left([0, T], H^{s}\right)}+\left\|v_{e}^{\eta}\right\|_{L^{\infty}\left([0, T], H^{s}\right)} \leq C_{0}
$$

\section{$A$ is skew symmetric}

Next $A$ is skew symmetric for the scalar product

$$
\left\langle\left(n_{1}, v_{1}\right),\left(n_{2}, v_{2}\right)\right\rangle=\int n_{e}^{0} v_{1} v_{2}+\frac{1}{n_{e}^{0}} n_{1} n_{2}+\nabla \phi_{1} \cdot \nabla \phi_{2}
$$

where $\phi_{1}=\Delta^{-1} n_{1}$ and $\phi_{2}=\Delta^{-1} n_{2}$. As a consequence $A$ has a basis of eigenvalues $\lambda_{j}\left(n_{e}^{0}\right)$ and of eigenspaces $\Pi_{j}\left(n_{e}^{0}\right)$. Note that this scalar product depends on $n_{e}^{0}$ and hence change with time, which add numerous technical difficulties to the computations.

The kernel of $A$ is

$$
\operatorname{ker}\left(A\left(n_{e}^{0}\right)\right)=\left\{(n, v) \quad \mid \quad \nabla \cdot\left(n_{e}^{0} v\right)=0,-\nabla\left(\frac{n}{n_{e}^{0}}\right)+\nabla \Delta^{-1} n=0\right\} .
$$

Note that the kernel of $A$ itself depends in a non obvious way from $n_{e}^{0}$.

Spectral analysis

First if $\lambda_{j}\left(n_{e}^{0}\right)$ is a simple eigenvalue, then it is locally differentiable and

$$
d \lambda_{j}\left(n_{e}^{0}\right) \tilde{n}=\int \Psi_{j}\left(n_{e}^{0}\right) \tilde{n}
$$

with

Next we define the resonant set by

$$
\Psi_{j}\left(n_{e}^{0}\right)=i \lambda_{j}\left(\frac{\left|n_{j}\right|^{2}}{\left(n_{e}^{0}\right)^{2}}-\left|u_{j}\right|^{2}\right)
$$

$$
\Sigma_{j, k, l}=\left\{\lambda_{j}=\lambda_{k}+\lambda_{l}\right\}
$$

Note that

$$
d\left(\lambda_{j}-\lambda_{k}-\lambda_{l}\right) \cdot \tilde{n}=\int\left(\Psi_{j}-\Psi_{k}-\Psi_{l}\right) \tilde{n}
$$

hence under the assumption

$$
(H 2) \quad \Psi_{j} \neq \Psi_{k}+\Psi_{l}
$$

$\Sigma_{j, k, l}$ is locally a smooth codimension 1 manifold. Again $(H 2)$ is as spectral assumption. We have no idea whether this assumption is generic or not. As previously we introduce the double eigenvalues

$$
\Sigma_{j, k}=\left\{\lambda_{j}=\lambda_{k}\right\} .
$$

If $n_{e}^{0} \in \Sigma_{j, k}$, if $\lambda_{j}$ and $\lambda_{k}$ are simple near $S_{0}$ and not triple at $S_{0}$, then a local analysis gives that $\Sigma_{j, k}$ is locally a codimension 2 manifold provided

$(H 1) \quad l_{j, k}^{1}$ and $l_{j, k}^{2}$ are linearly independent,

where

and

$$
l_{j, k}^{1}(\tilde{n})=<(d A . \tilde{n}) \sigma_{j}, \sigma_{j}>-<(d A . \tilde{n}) \sigma_{k}, \sigma_{k}>
$$

$$
l_{j, k}^{2}(\tilde{n})=<(d A . \tilde{n}) \sigma_{j}, \sigma_{k}>,
$$


$\sigma_{j}$ and $\sigma_{k}$ being eigenvectors associated with $\lambda_{j}$ and $\lambda_{k}$. Again $(H 1)$ is a difficult spectral problem

\section{Conclusion}

In this case the limit problem is smooth and well posed. Hence for almost every any initial data $n_{e}^{0}(t)$ avoids $\Sigma_{2}$ and crosses transversally $\Sigma_{3}$. As a consequence the resolvant of the wave equation is uniformly bounded as $\eta$ goes to 0 . It is possible to justify the formal limit (see [8]).

\section{REFERENCES}

[1] T. Alazard: Incompressible limit of the nonisentropic Euler equations with solid wall boundary conditions, Adv. in Differential Equations 10 (2005), $19-44$.

[2] J.-Y. Chemin, B. Desjardins, I. Gallagher, E. Grenier: Basics in Mathematical Geophysics, Oxford University Press

[3] B. Desjardins, E. Grenier: Low Mach number limit of viscous compressible flows in the whole space, R. Soc. Lond. Proc. Ser. A Math. Phys. Eng. Sci 455 (1999) $2271-2279$

[4] B. Desjardins, E. Grenier, P.-L. Lions, N. Masmoudi: Incompressible limit for solutions of the isentropic Navier Stokes equations with Dirichlet boundary conditions, J. Math. Pures Appl. 78 (1999), $461-471$.

[5] I. Gallagher: Résultats récents sur la limite incompressible, Séminaire Bourbaki 2003 - 2004, num 926.

[6] E. Grenier: Oscillatory perturbations of the Navier Stokes equations, Journal de Math. Pures et App. 76 (1997), 477 - 498.

[7] E. Grenier, Y. Guo, B. Pausader: Existence results for the massless limit in plasma, preprint 2011.

[8] E. Grenier, Y. Guo, B. Pausader: On the massless limit in plasma, preprint, 2011.

[9] S. Klainerman, A. Majda: Singular limits of quasilinear hyperbolic systems with large parameters and the incompressible limit of compressible fluids, Comm. Pure Appl. Math 34 (1981) $481-524$.

[10] S. Klainerman, A. Majda: Compressible and incompressible fluids, Comm. Pure Appl. Math 35 (1982, 629 - 651.

[11] G. Métivier, S. Schochet: Averaging theorems for conservative systems and the weakly compressible Euler equations, J. Differential Equations 187 (2003), 106 - 183.

[12] G. Métivier, S. Schochet: The incompressible limit of the non isentropic Euler equations, Arch. Ration. Mech. Anal. 158 (2001) $61-90$.

[13] S. Schochet: Fast singular limits of hyperbolic PDEs, J. Differential Equation 114 (1994), 476 - 512.

[14] S. Schochet: The mathematical theory of low Mach number flows, M2AN Math. Model. Numer. Anal., special issue, may june 2005.

[15] S. Ukai: The incompressible limit and the initial layer of the compressible Euler equation, J. Math. Kyoto Univ. 26 (1986, $323-331$. 